\title{
Análisis del impacto de la pandemia COVID-19 sobre la calidad de los servicios de salud sexual y reproductiva
}

\author{
Leonel Briozzo*, Fernanda Nozar ${ }^{\dagger}$, Verónica Fiol`, Cecilia Stapf§, \\ Sebastián Ben, Estela Citrin־, Diego Greif, Verónica Gallino**
}

\section{Resumen}

Antecedentes: la pandemia COVID-19 tiene el potencial de impactar fuertemente en la agenda de la salud y los derechos sexuales y reproductivos desde la disminución de la calidad de los servicios de salud.

Justificación: Uruguay se destaca por una situación privilegiada en lo referido a los derechos sexuales y reproductivos y por tal motivo es necesario, por un lado, sistematizar el impacto de la pandemia COVID-19 y, por otro, desarrollar medidas para prevenir dicho impacto sobre los servicios para mantener el más alto estándar de cuidados en lo relacionado a los derechos sexuales y reproductivos a pesar de la pandemia actual.

Propósito: sistematizar el potencial impacto de la pandemia en los servicios de atención sanitaria de salud sexual y reproductiva y promover estrategias para detectar y prevenir dicho impacto.

Metodología: se aborda el impacto de la pandemia COVID-19 sobre los derechos sexuales y reproductivos en dos dimensiones: la del modelo de retrasos en la atención de salud y la del análisis del impacto en cada derecho específico.

Perspectivas: promover acciones de discriminación positiva desde los servicios de salud sexual y reproductiva a la vez que se realiza la vigilancia epidemiológica para facilitar el acoplamiento de las prestaciones en derechos sexuales y reproductivos a los cambios que se procesan en la respuesta sanitaria y social frente a la pandemia COVID-19.

Palabras clave: COVID-19

Infecciones por coronavirus

Salud sexual y reproductiva

Salud de la mujer

Key words: $\quad$ COVID-19

Coronavirus infections

Sexual and reproductive health

Women's health

\footnotetext{
* Profesor. Clínica Ginecotocológica A. Facultad de Medicina, Universidad de la República.

† Ex Profesora Adjunta. Clínica Ginecotocológica A. Facultad de Medicina, Universidad de la República.

‡ Profesor Adjunto. Clínica Ginecotocológica A. Facultad de Medicina, Universidad de la República.

$\S$ Licenciada en Psicología. Iniciativas Sanitarias.

II Médico colaborador honorario. Clínica Ginecotocológica A. Facultad de Medicina, Universidad de la República.

** Asistente. Clínica Ginecotocológica A. Facultad de Medicina, Universidad de la República.

Correspondencia: Dra. Fernanda Nozar. Correo electrónico: fernandanozar@gmail.com

Recibido: $17 / 5 / 20$

Aprobado: $23 / 9 / 20$
} 


\section{Antecedentes}

La pandemia COVID-19 ha eclipsado, por su avance e impacto sanitario, una serie de problemas de salud, sobre todo los vinculados a la salud de la mujer ${ }^{(1)}$ y de los derechos sexuales y reproductivos ${ }^{(2)}$, especialmente en los sectores sociales más vulnerados en sus derechos humanos en relación con la pobreza y la desigualdad ${ }^{(3)}$. Estas situaciones se ven agravadas por el modelo hegemónico patriarcal de las relaciones sociales ${ }^{(4)}$, que no solo es riesgoso para las mujeres, sino también para la infancia y para los propios varones al incrementar conductas de riesgo, como la violencia interpersonal y el consumo problemático de sustancias psicoactivas. A esto se suma, en pandemia, un aumento de la presión derivada de la adversidad económica ocasionada por el brote y la incapacidad para trabajar y "abastecer", lo que puede llevar a una situación que provoca aún más tensiones y conflictos en el núcleo familiar, derivando en un aumento demostrado de la violencia basada en género, por ejemplo ${ }^{(5)}$.

Los cuatro principios generales desde el sistema sanitario de respuesta a la pandemia son: disminuir la velocidad de contagio en la comunidad mediante el distanciamiento social, aumentar la capacidad de realización de testeo PCR COVID-19, mejorar el funcionamiento y respuesta del Sistema Nacional Integrado de Salud (SNIS) en cuanto a la institucionalidad, los equipos, el primer nivel de atención, la atención prehospitalaria, la hospitalización, los cuidados intensivos, la telemedicina y, en cuarto lugar, incrementar las políticas sociales y la intersectorialidad, interinstitucionalidad y la inclusión financiera ${ }^{(6)}$.

Debe tenerse en cuenta, además, que un antecedente relevante que no se puede perder de vista es que el riesgo mayor en una pandemia como la actual es para los equipos de salud y cuidados. La mayoría de los integrantes de estos equipos son mujeres, calculándose en un $70 \%$ su número, lo que las expone a un riesgo superlativo en una situación como la actual.

Por último, la pandemia de COVID-19 se puede enmarcar en lo que se denomina sindemia global, que es la suma y potenciación de las tres epidemias más importantes en la sociedad contemporánea, que tienen causas y mecanismos de desarrollo comunes, a saber: el cambio climático, la malnutrición y la obesidad ${ }^{(7)}$. En última instancia, los circuitos causales de la pandemia COVID-19 y de la sindemia global se relacionan a un mismo fenómeno: el modelo de producción agrícola intensivo destructor del medio ambiente que impone la economía de mercado contemporánea y que hace, entre otras muchas cosas, que reservorios animales entren en contacto con humanos, promoviendo eventualmente con intermedia- rios animales de criaderos ultraintensivos el pasaje y contagio del humano por nuevos tipos virales ${ }^{(8)}$. Frente a esta situación, la visión que integra la salud con el medio ambiente, denominada una sola salud ${ }^{(9)}$, debe imponerse en cualquier análisis unilateral que aborde la salud solo tomando en cuenta lo que ocurre en la intimidad de la relación médico-paciente-sistema de salud.

\section{Justificación}

Uruguay es reconocido internacionalmente en la agenda global del cumplimiento de los derechos sexuales y reproductivos, siendo esto reflejado en indicadores específicos cualitativos y cuantitativos de acuerdo con los seis derechos básicos. Los principales indicadores se expresan en cada derecho sistematizadamente.

El primer derecho analizado es el ejercicio de la maternidad sin riesgos innecesarios de enfermedad y muerte, a propósito del cual se analiza: la mortalidad materna, la mortalidad infantil, la prevalencia de prematurez, la transmisión vertical de VIH y sífilis.

- Mortalidad materna: la tasa de mortalidad materna en Uruguay ha disminuido significativamente en los últimos 25 años, presentando en la actualidad la tasa más baja de mortalidad materna en las Américas, justo detrás de Canadá, con 11 muertes cada 100.000 recién nacidos vivos ${ }^{(10)}$. La reducción se debe fundamentalmente a la disminución del aborto inseguro (responsable de casi el $40 \%$ de las muertes maternas al comienzo de la década del 2000) en el contexto de la promoción de todos los derechos sexuales y reproductivos y el aseguramiento de las prestaciones sanitarias.

- Mortalidad infantil: en el año 2019 se registraron 2.671 nacimientos menos y 14 defunciones menos en menores de 1 año en relación con 2018. La tasa de mortalidad infantil fue de 6,8/1.000 recién nacidos vivos, tasa igual a la de 2018, manteniendo la tendencia decreciente de los últimos 15 años ${ }^{(11)}$.

- Prematurez: el porcentaje de parto prematuro en Uruguay se mantiene con valores estables, cercanos al 9\% de los nacimientos. En 2018, el porcentaje de prematurez a nivel nacional fue de $9,2 \%$ de los nacimientos, siendo a nivel público de $9,3 \%$ y en el subsector privado de 9,1\%. En 2018, en el Hospital de la Mujer del Centro Hospitalario Pereira Rossell, el $11,8 \%$ de los nacimientos fueron prematuros, manteniéndose estable en los últimos cinco años. La importancia de la prematurez radica en que se trata de una de las principales causas de morbilidad y mortalidad infantil.

- Transmisión vertical de VIH y sífilis: se lograron avances significativos hacia el cumplimiento de las 
metas de eliminación de VIH ( $\leq 2 \%)$, que se alcanzó en los últimos tres años, mientras que se ha avanzado hacia el logro de la meta de eliminación de la sífilis congénita $(\leq 0,5$ casos $/ 1.000$ recién nacidos vivos), alcanzándose ya en el subsector privado.

El segundo derecho es el que se refiere al control individual de la propia fertilidad de la persona. A este respecto los indicadores más importantes se refieren a la maternidad-paternidad responsable, la fertilidad en la población adolescente y el derecho reconocido a la reproducción asistida.

- Maternidad-paternidad responsable y segura. La anticoncepción es una prestación del SNIS, existiendo una canasta de métodos que obligatoriamente los prestadores de salud deben brindar a sus usuarios. La misma incluye anticoncepción hormonal, dispositivo intrauterino, preservativos, ligadura tubaria y vasectomía (Decreto 009/11). El acceso a métodos anticonceptivos está regulado por la Ley 18.426 de Salud Sexual y Reproductiva, artículo 3, y su decreto reglamentario, que obliga a la entrega de píldoras anticonceptivas de cuatro tipos con tiques reguladores muy accesibles.

- La estrategia intersectorial y nacional de prevención del embarazo no intencional en adolescentes, en conjunto con las acciones realizadas desde las políticas sanitarias, logró reducir la tasa de embarazos en $38 \%$ en 2018. Disminución del embarazo en adolescentes de 15 a 19 años de 16,4\% en 2014 a 11,3\% en $2018 ; 10,6 \%$ en $2019^{(11)}$.

- Reproducción humana asistida: en 2013, la Ley 19.167 de Técnicas de Reproducción Humana Asistida (RHA) estableció el derecho de las parejas biológicamente impedidas, y de las mujeres independientemente de su estado civil, a acceder a técnicas de reproducción asistida, incorporando su diagnóstico y tratamiento a las prestaciones que se brindan en el SNIS. En el año 2019 se registran 676 solicitudes al Fondo Nacional de Recursos, se autorizó el $87,28 \%$. En 2018, 622 aprobadas $(88,1 \%)$ y 739 en 2017, autorizadas el 91,07\%.

El tercer derecho se refiere a la posibilidad de tener una vida sexual libre de violencia, coerción o riesgo de adquirir enfermedad y embarazo no deseado.

- Violencia basada en género (VBG): este derecho en Uruguay está severamente amenazado por una tasa de femicidios alarmante. Uruguay es uno de los países de América Latina que tiene mayor cantidad de muertes de mujeres ocasionadas por parejas o exparejas cada 100.000 mujeres. En Uruguay, una de cada cinco mujeres vivió algún episodio de VBG en los últimos 12 meses por parte de algún integrante de la familia. En las mujeres que tienen o han tenido pareja la prevalencia de VBG es de 45,5\% en algún momento de su vida, especialmente en las pacientes jóvenes. El año 2019 se cerró con una declaración de emergencia nacional por violencia de género, registrándose 25 femicidios.

- Diversidad: en 2018, Uruguay aprobó la Ley 19.684, Integral para personas trans, garantizando el acceso a derechos en relación con la identidad, el trabajo, la educación, la reparación histórica a víctimas del terrorismo de Estado y a la salud libre de discriminación. En los servicios de salud, establece que el 57\% de las instituciones mejoraron el cumplimiento con personal formado en diversidad sexual, pasaron de $17 \%$ en 2016 a 49\% en 2018. El 43\% de los prestadores respeta la identidad de género de sus usuarios/as en la asignación de camas. Los prestadores que brindan tratamientos hormonales pasaron de $26 \%$ en 2016 a $53 \%$ y los quirúrgicos de $8 \%$ a $15 \%$.

El cuarto derecho involucrado es el que se refiere a la interrupción del embarazo en los casos legalmente autorizados o admitidos por el sistema jurídico. Desde hace más de 20 años, Uruguay implementó un modelo original de reducción de riesgo y daños desarrollado por la asociación civil Iniciativas Sanitarias. Esta estrategia, junto con el mejoramiento del acceso a los métodos anticonceptivos y la educación en salud sexual y reproductiva, resultó clave en la reducción de la mortalidad materna ${ }^{(11)}$. Luego de siete años de vigencia de la ley de despenalización del aborto, con la consiguiente implementación de servicios de atención en todo el SNIS, así como de una mejora sistemática de la fiscalización y el registro, en el año 2019 se contabilizaron 10.227 casos de interrupciones voluntarias de embarazo (IVE), registrándose 146 menos que el año previo. Este último hecho resulta relevante, ya que es la primera vez que la curva comienza a mostrar una tendencia decreciente en el número total de IVE. Las IVE registradas en 2019 contabilizaron 10.227 casos*, siendo 146 menos que el año, siendo este el primer año en que descendieron las cifras desde la despenalización del aborto ${ }^{(11)}$. El mantenimiento de este descenso es un objetivo importante de la implementación de la política de despenalización del aborto en Uruguay, ya que, como oportunamente se planteó, de lo que se trata es de asegurar una práctica segura, accesible e infrecuente ${ }^{(11)}$.

El quinto derecho sexual y reproductivo se refiere a la disponibilidad de servicios para el ejercicio de esos derechos. Uruguay ha cumplido cabalmente con este derecho asegurando los servicios de salud sexual y repro- 
ductiva con la base de la Ley 18.426 de Defensa del Derecho a la Salud Sexual y Reproductiva, desde la cual el Ministerio de Salud Pública estableció la obligatoriedad de implementar servicios de salud sexual y reproductiva en todos los prestadores de salud del SNIS.

Por último, el sexto derecho se refiere a la necesidad de información a partir de los derechos involucrados y los servicios adecuados. Al respecto, la educación sexual está presente en todos los niveles de la enseñanza formal a partir de 2006. Desde 2011, se crea una comisión interinstitucional para la promoción de los derechos sexuales y reproductivos, promoviendo acciones conjuntas de salud, educación y desarrollo social en todo el sistema educativo formal, como parte de la formación hacia una ciudadanía plena.

En suma: Uruguay tiene un alto estándar en el cumplimiento de las prestaciones sanitarias que aseguran los derechos sexuales y reproductivos, y la pandemia COVID-19 puede representar una amenaza a las estrategias desarrolladas, a los logros alcanzados y en los indicadores que reflejan la situación nacional privilegiada a este respecto.

\section{Propósito}

Sistematizar el potencial impacto de la pandemia COVID-19 en los servicios de atención sanitaria de salud sexual y reproductiva y promover estrategias para detectar y prevenir dicho impacto.

\section{Metodología}

Para sistematizar los probables riesgos de la pandemia se analiza, por un lado, los retrasos y las barreras para la atención en salud sexual y reproductiva y, por otro, el probable impacto en cada derecho.

$\mathrm{Al}$ abordar específicamente la salud de la mujer en lo relacionado a los derechos sexuales y reproductivos, así como en la consideración de los retrasos en la mortalidad materna ${ }^{(11)}$, se puede hacer referencia a los retrasos que existen en la atención de salud a las mujeres embarazadas que los requieren. Así, el primer capítulo de estudio se organiza analizando el nivel de retraso en la definición de la necesidad de consultar; el nivel del retraso en el traslado; el nivel del retraso en el acceso a los servicios, y el nivel de retraso en la provisión de la atención por parte del sistema sanitario.

\section{Retrasos y barreras para la atención en salud sexual y reproductiva}

1. Nivel de retraso en definir la necesidad de consultar: la identificación y valoración de síntomas o motivos para consultar están en estos momentos opacadas por la gravedad epidemiológica y el pánico social que genera la pandemia.
El cierre de escuelas para controlar la transmisión de la COVID-19 tiene un efecto económico diferencial en las mujeres, ya que ellas se ocupan de la mayor parte del cuidado informal al interior de las familias, con consecuencias que limitan sus oportunidades laborales y económicas. En general, la experiencia de un brote también significa que la carga doméstica para las mujeres se verá exacerbada, considerando que la proporción de sus responsabilidades en el hogar aumenta, y, en el caso de muchas de ellas, al mismo tiempo que trabajan en forma de tiempo completo.

2. Nivel de retraso en el traslado desde el domicilio al centro de atención: existen dificultades de transporte en relación con el aislamiento social y la disminución del transporte público que pueden provocar retrasos en la atención de la salud. Por tal motivo, es relevante que los transportes sanitarios, cuando hay motivos de consulta relacionados con la problemática, estén asegurados.

3. Nivel de retraso en el acceso a los servicios de salud: existe menos disponibilidad de acceso al primer nivel de atención y acceso limitado al segundo y tercer nivel dada la recomendación de consultar. Asimismo, el sistema de consulta domiciliario no está preparado en general para el abordaje de problemas relacionados con la salud sexual y reproductiva. Aquí surge un problema peculiar y es que la promoción de la telemedicina, muy útil para varios aspectos, no lo es para muchos de los temas vinculados con la salud sexual y reproductiva, $y$, con la idea errónea de que hay servicios que se pueden brindar por telemedicina, se puede diferir una necesaria consulta en persona, como, por ejemplo, en casos de metrorragias gravídicas, hidrorrea, percepción de disminución de movimientos fetales, etc. Concomitantemente, las consultas remotas son muy vulnerables desde el punto de vista de la confidencialidad y por los peculiares motivos de consulta, por ejemplo, embarazo no deseado, anticoncepción adolescente, violencia, etc.

4. Nivel de retraso en la provisión de la atención pertinente del SNIS: los servicios pueden estar disminuidos por falta de servicios habilitados (por ejemplo, servicios de salud sexual y reproductiva) e inclusive de stock insuficiente.

Información pública: en el contexto de la alerta social, provocada por un lado por la amenaza real de la infección, pero sobre todo por la confusión por la variabilidad, por la procedencia, por diferentes intereses, por la propia complejidad en la interpretación del mensaje "importa tanto la salud pero no puedo 
concurrir", todo conspira en favor de la confusión y la dificultad en las definiciones.

\section{Impacto particular en cada derecho sexual y reproductivo}

La pandemia COVID-19 afecta de manera específica a cada uno de los derechos sexuales y reproductivos y lo hace de manera diferencial de acuerdo con la sumatoria de otras vulnerabilidades vinculadas sobre todo con la pobreza y la exclusión, que se verá agravada indudablemente por la presente pandemia. Más allá de esto, analizando específicamente la matriz de cada derecho, se puede evaluar:

1. Impacto de la pandemia COVID-19 en el ejercicio de la maternidad sin riesgos innecesarios de enfermedad y muerte.

- Por la infección en sí misma: no existen evidencias de que el SARS-CoV-2 provoque afección particular en las mujeres durante el embarazo ${ }^{(11)}$. Existen sí reportes de incremento de la prematurez vinculada con la posibilidad de insuficiencia respiratoria y de restricción de crecimiento fetal por la afectación placentaria $^{(11)}$.

- Por los cambios en los sistemas de salud y de los cuidados (protección personal de los equipos de salud): afectará en una disminución de los controles de embarazos, de acontecer, llevará a un aumento de la patología materna perinatal no diagnosticada, además del impacto en la calidad y humanización de los cuidados ${ }^{(11)}$. Es claro que a pesar de los esfuerzos de los equipos de salud, aumente el intervencionismo innecesario desde el punto de vista obstétrico y por tanto la iatrogenia en estas pacientes.

- El derecho al acompañamiento en el parto, que en Uruguay está amparado por la Ley 17386 desde el año 2001, también se encuentra en riesgo. Si bien las recomendaciones internacionales plantean limitar los acompañantes en caso de mujeres infectadas por COVID-19, muchas instituciones de salud en Uruguay limitan o incluso impiden el acompañamiento durante el parto o cesárea en mujeres asintomáticas, en pro de la protección del personal de salud y la preservación de insumos materiales.

- Desde un punto de vista teórico la pandemia aumenta la vulnerabilidad en los sectores ya vulnerados en sus derechos y, por tanto, incrementa el riesgo de desarrollo de los dos grandes síndromes obstétricos perinatales, a saber, la restricción de crecimiento fetal y la prematurez ${ }^{(11)}, y$, por ende, es dable esperar un aumento del efecto de éstos en la reproducción transgeneracional de la pobreza y en las enfermedades crónicas por la activación epigenética ${ }^{(11)}$.
2. Impacto de la pandemia COVID-19 en el control individual de su propia fertilidad.

- La falta de valoración de la contracepción como servicio esencial amenaza la continuidad de los servicios que potencia el retraso de consulta y las dificultades para el acceso a los servicios.

- Disponibilidad de insumos y cadenas de abastecimiento pueden verse amenazadas, sobre todo de los métodos más idóneos durante una pandemia como son los de larga duración.

3. Impacto de la pandemia COVID-19 en una vida sexual libre de violencia, coerción o riesgo de adquirir enfermedad y embarazo no deseado.

- El aislamiento y la reclusión aumenta la VBG, como lo demuestran las evidencias disponibles de incremento del feminicidio. En épocas de crisis, como sucede durante un brote, las mujeres y las niñas pueden presentar un riesgo más elevado, por ejemplo, de padecer violencia infligida por la pareja y otras formas de violencia intrafamiliar como resultado de las tensiones crecientes en el hogar. También enfrentan mayores riesgos de otras formas de violencia de género, incluidas la explotación y el abuso sexual en estas situaciones. Adicionalmente, la atención para salvar vidas y el acompañamiento a las sobrevivientes de violencia de género (es decir, el manejo clínico de la atención en casos de violación y el apoyo a la salud mental y psicosocial) pueden reducirse como parte de la respuesta de atención de la salud si los prestadores de servicios enfrentan una carga de trabajo excesiva y una mayor preocupación por el manejo de los casos de COVID-19.

- Dificultades de relacionamiento interpersonal por miedo y desinformación vinculada con distanciamiento social e incremento de las disfunciones sexuales. No hay aún estudios que evalúen el impacto de la pandemia en las disfunciones preexistentes en tratamiento o la aparición de nuevas alteraciones de la esfera sexual; sin embargo, es lógico pensar que disfunciones no resueltas o en tratamiento pueden ver demorada su resolución, recaer o empeorar de la misma manera que otras patologías. Es interesante mencionar que el aspecto relacional se encuentra especialmente desafiado en esta pandemia, sobre todo si la relación previa se encontraba en una situación inestable.

- Aumento de embarazos no deseados e interrupción voluntaria del embarazo. Los embarazos no deseados pueden aumentar por varias razones: falta de acceso a servicios de anticoncepción; aumento de las situaciones de violencia basada en género y abuso sexual; empeoramiento de la situación socioeconómica. 
4. Impacto de la pandemia COVID-19 en el derecho a la interrupción del embarazo en los casos legalmente autorizados o admitidos por el sistema jurídico.

- Los retrasos en el acceso a servicios vinculados con la disminución de las consultas puede aumentar las captaciones más allá de las 12 semanas de edad gestacional, lo que puede aumentar el riesgo de los abortos voluntarios.

- El aumento de la telemedicina y la disminución de la confidencialidad incrementó el riesgo para las poblaciones más vulneradas en sus derechos. Disminuye la confianza en el sistema y aumenta el riesgo. Esto determina muchas veces la autoexclusión del sistema de salud.

5. Impacto de la pandemia COVID-19 en la disponibilidad de servicios para el ejercicio de esos derechos.

- Los servicios de salud sexual y reproductiva pueden disminuir, ya que el modelo médico hegemónico no lo considera esencial. La respuesta de emergencia al brote de COVID-19 en gran medida significa que los recursos económicos, de gestión, profesionales y materiales para los servicios de salud sexual y reproductiva pueden desviarse con el propósito de hacer frente al brote.

- La provisión de insumos de planificación familiar y otros insumos de salud sexual y reproductiva, incluidos artículos para la salud menstrual, es central para la salud, el empoderamiento y el desarrollo sostenible de las mujeres, y puede verse afectada cuando las cadenas de suministros se ven sometidas a alteraciones como resultado de la respuesta a la pandemia.

6. Impacto de la pandemia COVID-19 en la información sobre sus derechos y los servicios que los aseguren.

- Los derechos sexuales y reproductivos son opacados por la paranoia infectológica informativa relacionada al COVID-19 y, por tanto, puede hacer que los derechos como tales sean invisibilizados.

- Retroceso en el empoderamiento con respecto a los derechos impulsados desde sectores conservadores.

\section{Perspectivas}

Las evidencias han mostrado que la adecuación legislativa, sanitaria y de recursos ha sido determinante en la disminución de la mortalidad materna y neonatal, en el marco de la integralidad de promoción de los derechos sexuales y reproductivos como derechos humanos en Uruguay. Por todo lo analizado anteriormente, y de no tomarse medidas que aseguren las prestaciones de estos derechos, es un riesgo real que ocurra un aumento en la mortalidad materna y neonatal, un incremento en las necesidades insatisfechas de planificación familiar y un aumento en el número de abortos en condiciones de riesgo e infecciones de transmisión sexual, como indicadores de vulneración de derechos.

Se debe enfocar los esfuerzos en:

- potenciar la salud sexual y reproductiva,

- optimizar el control del embarazo y la atención del nacimiento,

- mejorar la performance de los equipos de salud sexual y reproductiva, los del primer nivel de atención y los de asistencia prehospitalaria.

En los servicios: innovar mediante telemedicina, servicios digitales, clínicas móviles y preparación específica de los equipos de salud. Como plantea la Federación Internacional de Ginecología y Obstetricia (FIGO): "FIGO apoya la provisión de servicios sostenibles y tiene el compromiso de servir a las mujeres de manera segura mientras navegamos por estas aguas desconocidas y pedimos a los gobiernos que hagan lo mismo. Los cambios deben implementarse rápidamente para salvar vidas y evitar una mayor presión sobre los servicios médicos. Pero necesitamos cambios duraderos que prevengan a las mujeres de tener que buscar métodos insegu-

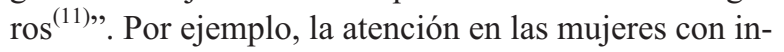
tención de IVE no puede verse alterada ni retrasada porque, como está demostrado, puede aumentar la mortalidad materna y también la morbilidad grave, que puede tener severas consecuencias en la mujer y sus hijos. En aquellas mujeres que manifiestan deseo de IVE que no están dentro del marco legal, se debe realizar el correcto asesoramiento para que la interrupción no sea en condiciones de riesgo. Se debe garantizar la continuidad de la atención para las mujeres y las niñas en edad reproductiva en caso de presentarse una interrupción o alteración severa de los servicios en las instalaciones. Es necesario hacer frente a los obstáculos y las barreras a través de acciones para facilitar el acceso de las mujeres y las niñas a los servicios, incluidos servicios de apoyo psicosocial, especialmente para aquellas víctimas de violencia o que pudieran estar en riesgo de experimentar violencia durante una cuarentena.

En los cuidados obstétricos es conveniente adoptar esquemas adecuados de control, por ejemplo, para las mujeres de bajo riesgo. Un esquema razonable sería ver al paciente en cada trimestre, cronometrado con las pruebas programadas. Por ejemplo, entre la 11 y 14 semanas para una valoración morfoestructural precoz, cribado genético y primeras rutinas; entre las 20 y 24 semanas para el escaneo y revisión de la morfología y segundas rutinas; 28 semanas para el cribado de diabetes gestacional, la administración de inmunoglobulina anti-D, si corresponde, y administración de vacuna dpTa, y a las 36 semanas para ruti- 
nas del tercer trimestre y búsqueda de estreptococo del grupo B y 38 semanas. A medida que la pandemia se expande, se debe considerar incluso menos visitas. Cuando sea práctico, las citas deben realizarse por teléfono o con videoconferencia, según corresponda, para el seguimiento de la comorbilidad médica. Las mujeres con síntomas de COVID-19 deben hacerse la prueba y retrasar una cita, si es posible, durante el período de autocuarentena. Si los síntomas persisten, deben llamar y hacer una cita para la prueba o la hospitalización. Los avances en telesalud y monitoreo remoto hacen que las visitas virtuales sean una realidad en algunos lugares. Las medidas adicionales deben incluir limitar el número de personas de apoyo/visitantes con pacientes para visitas ambulatorias y hospitalarias, incluidas las áreas de parto y parto ${ }^{(11)}$. Con respecto al nacimiento, el desafío principal es mantener los estándares asistenciales de acuerdo a la definición de promoción del parto humanizado e institucional en las maternidades para todas las usuarias (sin o con infección por COVID-19), manteniendo en todos sus términos los protocolos nacionales en la materia, a la vez que se desarrolla una política de protección total del personal y los equipos de salud. Esta protección debe interpretarse como una obligación bioética hacia las usuarias y la sociedad, en la medida que evitando el contagio en los equipos, se hará sostenible la atención para las pacientes actuales y futuras. Capítulo aparte merece la consideración a propósito de los acompañantes, por el momento no existe evidencia que contradiga el acompañamiento de la mujer en el nacimiento, determinándose un único acompañante elegido por la mujer que se encuentre asintomático y sin noción de contagio de COVID-19.

Con respecto a los equipos de salud, se debe asegurar que el personal de salud cuente con las habilidades y los recursos necesarios para manejar información sensible relacionada con la violencia de género, y ser propositivos en su indagatoria para que cualquier reporte sobre violencia de género sea tratado con respeto, empatía y confidencialidad, y que los servicios se proporcionen con un enfoque centrado en las sobrevivientes. También es fundamental actualizar las rutas de derivación para casos de violencia de género de modo que reflejen los cambios en las instalaciones de atención disponibles, además de informar a las comunidades y los prestadores de servicios clave acerca de la actualización de dichas rutas. En lo que respecta a la sexualidad y el placer sexual es importante generar un mensaje propositivo. Se replica la recomendación sobre aspectos vinculados a la sexualidad por parte del New York Health Department, que promueve la práctica de sexo virtual o masturbación en caso de no tener una pareja conviviente, brindando además recomendaciones sobre el uso de juguetes sexuales. Las recomendaciones de sociedades científicas hacen hincapié en la prevención del embarazo no inten- cional y de ITS, aunque no brindan herramientas para el desarrollo de la actividad sexual enfocadas a la búsqueda del placer.

Ensamblada la respuesta de derechos en el contexto de la respuesta global del SNIS, se debe desarrollar la herramienta de vigilancia epidemiológica para orientar las acciones. La evaluación del impacto se debe procesar en los sistemas de vigilancia y respuesta deben incluir datos desagregados por sexo, edad, género y estado de embarazo. Una perspectiva en este sentido podría ser que el sistema se prepare desde ya al registro y evaluación longitudinal de los resultados sanitarios. Es decir que el monitoreo debe ser sostenido y no transcurridos largos períodos.

La participación ciudadana y el empoderamiento de las mujeres es clave en la respuesta, ya que juegan un rol al interior de las comunidades, típicamente las coloca en una buena posición para influir de manera positiva en el diseño y la implementación de actividades de prevención. Dada su proximidad a nivel local, sus acciones de vigilancia y sus perspectivas pueden ayudar a detectar el inicio de un brote y mejorar la situación de la salud en general. Incorporar las voces de las mujeres en la primera línea de la respuesta a través de la inclusión de las mujeres que forman parte del personal de salud y aquellas más afectadas por la enfermedad en las políticas o prácticas de preparación y respuesta a futuro. Apoyar la participación significativa de las mujeres y las niñas a nivel comunitario, incluidas sus redes y organizaciones, para asegurarse de que los esfuerzos y la respuesta no continúen discriminando y excluyendo a las personas con mayor riesgo. Asegurar estrategias de trabajo comunitario con mujeres como una forma de fortalecer el autocuidado en salud sexual y reproductiva es una prioridad establecida por la Organización Mundial de la Salud. Sobre intervenciones, define el autocuidado como "la capacidad de las personas, familias y comunidades para promover la salud, prevenir enfermedades, mantener la salud y hacer frente a enfermedades y discapacidades con o sin el apoyo de un proveedor de servicios de salud ${ }^{(11)}$. Esto es especialmente relevante para avanzar en la salud y en los derechos sexuales y reproductivos de las mujeres y niñas afectadas por barreras culturales, sociales, religiosas, políticas y legales que limitan u obstaculizan el acceso a servicios de calidad, información apropiada, opciones, autonomía y agencia. Las intervenciones de autocuidado proporcionadas en el marco de un entorno seguro y de apoyo, con sistemas de salud responsables y políticas habilitadoras y en asociación con las comunidades afectadas, pueden dar lugar a la satisfacción de las necesidades y derechos incluso de las poblaciones más vulnerables $^{(11)}$. 
Por último, en el contexto de la pandemia, es clave prepararse para el futuro. Posiblemente y de no mediar un cambio dramático en los sistemas productivos destructores del medio ambiente, las pandemias llegaron para quedarse. Existen iniciativas globales, como la de solicitar el establecimiento de un Task Force de equidad en salud global para apoyar la respuesta a la pandemia de COVID-19 recibido por el secretario general de la Organización de Naciones Unidas, el 21 de abril de 2020, representando sus propuestas y contenidos actuales en el sitio web: www.sustainablehealthequity.org

Se debe fortalecer el rol de los Estados y más políticas públicas vinculadas a los fines centrales de éstos: la salud, la educación y la seguridad son y serán la clave, en conjunto con más ciencia, y la tecnología como iniciativa pública global, regional y nacional, ya que al decir de Roberto Caldeiro Barcia, la ciencia y la tecnología son la diferencia entre la libertad y la dependencia. Al respecto, es importante potenciar los ámbitos institucionales y crear una nueva institucionalidad de acción política-técnica-científica.

\section{Summary}

Background: the COVID 19 pandemic may severely influence the sexual and reproductive rights and health agenda as a result of a decrease in the health services.

Justification: Uruguay has a privileged situation in terms of sexual and reproductive rights. Therefore, we need to systematize and develop measures to prevent the impact of the covid-19 pandemic on these services in order to keep the highest standards of care in connection with sexual and reproductive rights, despite the current pandemic.

Objective: to systematize the potential impact of the covid-19 pandemic on sexual and reproductive rights and health services and to promote strategies to identify and prevent this impact.

Method: the impact of the covid-19 pandemic on sexual and reproductive rights is analysed from two perspectives: by considering the delay in the provision of health care services and by exploring its impact on each specific sexual and reproductive right.

Perspectives: to promote positive discrimination actions at the sexual and reproductive rights services, while conducting epidemiological surveillance to contribute to the matching of sexual and reproductive rights services to the changes caused by the covid-19 pandemic on the health system and on society.

\section{Resumo}

Antecedentes: a pandemia COVID-19 tem o potencial de impactar fortemente sobre a agenda da saúde e os di- reitos sexuais e reprodutivos a partir da queda na qualidade dos serviços de saúde.

Justificativa: o Uruguai se destaca por uma situação privilegiada em matéria de direitos sexuais e reprodutivos (DDSSRR) e por isso é necessário, por um lado, sistematizar o impacto da pandemia COVID-19 e por outro, desenvolver medidas para prevenir esse impacto sobre os serviços para manter o mais alto padrão de atendimento em relação aos direitos sexuais e reprodutivos, apesar da pandemia atual.

Objetivo: sistematizar o impacto potencial da pandemia COVID-19 nos serviços de saúde sexual e reprodutiva e promover estratégias para detectá-lo e preveni-lo.

Metodologia: o impacto da pandemia COVID-19 sobre os DDSSRR é abordado em duas dimensões: a do modelo de atrasos na assistência de saúde e a da análise do impacto em cada um dos DDSSRR.

Perspectivas: promover ações de discriminação positiva dos serviços de saúde sexual e reprodutiva, enquanto a vigilância epidemiológica é feita para facilitar o acoplamento dos benefícios dos direitos sexuais e reprodutivos às mudanças que ocorrem na resposta sanitária e social a pandemia COVID19.

\section{Bibliografía}

1. Wenham C, Smith J, Morgan R; Gender and COVID-19 Working Group. COVID-19: the gendered impacts of the outbreak. Lancet 2020; 395(10227):846-8. Disponible en: https://www.thelancet.com/journals/lancet/article/PIIS01406736(20)30526-2/fulltext [Consulta: 16 febrero 2020].

2. Fondo de Población de las Naciones Unidas. Covid-19: un enfoque de género. Proteger la salud y los derechos sexuales y reproductivos y promover la igualdad de género. (Informe técnico) marzo 2020. Disponible en: https://uruguay.unfpa.org/sites/default/files/pub-pdf/COVID-

19\%20A\%20Gender\%20Lens\%20Guidance\%20Note.docx_en-US_es-MX_0.pdf [Consulta: 12 abril 2020].

3. Starrs AM, Ezeh AC, Barker G, Basu A, Bertrand JT, Blum R, et al. Accelerate progress-sexual and reproductive health and rights for all: report of the Guttmacher-Lancet Commission. Lancet 2018; 391(10140):2642-92. doi: 10.1016/S0140-6736(18)30293-9.

4. Carter J. Patriarchy and violence against women and girls. Lancet 2015; 585(9978):e40-1. doi: 10.1016/S0140- 6736 (14)62217-0.

5. UNFPA; Avenir Health, Johns Hopkins University; Victoria University (Australia). Impact of the COVID-19 pandemic on family planning and ending gender-based violence, female genital mutilation and child marriage. UNFPA, 2020.

6. Briozzo L. Origen, impacto sanitario y perspectivas de la Pandemia COVID 19. La Diaria 9 abril 2020. Disponible en: https://adiaria.com.uy/articulo/2020/4/origen-impacto-sani- 
tario-y-perspectivas-de-la-pandemia-covid-19/ [Consulta: 12 abril 2020].

7. Swinburn B, Kraak V, Allender S, Atkins V, Baker P, Bogard $\mathbf{J}$, et al. The global syndemic of obesity, undernutrition and climate change: the Lancet Commission report. Lancet 2019; 393:791-846.

8. Shah S. ¿De dónde viene el coronavirus? Le Monde Diplomatique 2020 marzo. Disponible en: https://www.lemondediplomatique.cl/2020/03/de-donde-viene-el-coronavirus.html [Consulta: 12 abril 2020].

9. Medina J. Pandemia por SARS-CoV-2 (COVID-19): entre la incertidumbre y la fortaleza. Rev Méd Urug 2020; 36(2):119-20

10. Briozzo L, Gómez Ponce de León R, Tomasso G, Faúndes A. Overall and abortion-related maternal mortality rates in Uruguay over the past 25 years and their association with policies and actions aimed at protecting women's rights. Int J Gynaecol Obstet 2016; 134:S20-3.

11. Uruguay. Ministerio de Salud Pública. Tendencias recientes de la natalidad, fecundidad y mortalidad infantil en Uruguay. Montevideo: MSP, 2020. Disponible en: http://www. presidencia.gub.uy/comunicacion/comunicacionnoticias/salud-embarazo-adolescente-mortalidad-infantil-bassomsp-cifras-fecundidad [Consulta: 26 abril 2020].

12. Briozzo L. From risk and harm reduction to decriminalizing abortion: the Uruguayan model for women's rights. Int J Gynaecol Obstet 2016; 134(Suppl 1):S3-6. doi: 10.1016/j.ijgo.2016.06.003.

13. Briozzo L. La despenalización del aborto como estrategia hacia una práctica segura, accesible e infrecuente. Rev Méd Urug 2013; 29(2):114-7.

14. Maine D, Rosenfeld A. The safe motherhood initiative: why has it stalled? Am J Public Health 1999; 89(4):480-2.

15. Fondo de Población de las Naciones Unidas. UNFPA statement on novel coronavirus (COVID-19) and pregnancy. UNFPA, 2020. Disponible en: https://www.unfpa.org/ press/unfpa-statement-novel-coronavirus-covid-19-and-pregnancy [Consulta: 12 abril 2020].
16. Wang X, Zhou Z, Zhang J, Zhu f, Tang $\mathbf{Y}$, Shen $\mathbf{X}$. A case of 2019 novel coronavirus in a pregnant woman with preterm delivery. Clinical Infect Dis 2020; 71(15):844-6. Disponible en: https://academic.oup.com/cid/advance-article/doi/10.1093/cid/ciaa200/5771323 [Consulta: 24 marzo 2020].

17. Poon LC, Yang H, Kapur A, Melamed N, Blami D, Divakar $\mathbf{H}$, et al. Global interim guidance on coronavirus disease 2019 (COVID-19) during pregnancy and puerperium from FIGO and allied partners: information for healthcare professionals. Int J Gynaecol Obstet 2020; 149(3):273-86. doi: 10.1002/IJGO.13156.

18. Briozzo L, Viroga S. Abordaje obstétrico integral de la prematurez y restricción del crecimiento fetal y su relación con las enfermedades crónicas del adulto. Rev Méd Urug 2020; 36(1):216-34.

19. Briozzo L, Coppola F, Gesuele JP, Tomasso G. Restricción de crecimiento fetal, epigenética y transmisión trans generacional de las enfermedades crónicas y la pobreza. Horiz Méd $2013 ; 13: 45-53$.

20. Federación Internacional de Ginecología y Obstetricia. Acceso al aborto y seguridad con COVID-19. FIGO, 30 mar 2020. Disponible en: https://www.figo.org/es/acceso-al-aborto-y-seguridad-con-covid-19 [Consulta: 26 abril 2020].

21. Federación Internacional de Ginecología y Obstetricia. Maternidad segura y COVID-19. FIGO, 30 mar 2020. Disponible en: https://www.figo.org/es/maternidad-segura-y-covid-19 [Consulta: 26 abril 2020].

22. World Health Organization. WHO consolidated guideline on self-care interventions for health: sexual and reproductive health and rights. Geneva: WHO, 2019. Disponible en: https://apps.who.int/iris/bitstream/handle/10665/325480/9789241550550-eng.pdf?ua $=1$ [Consulta: 16 febrero 2020].

23. Narasimhan M, Allotey P, Hardon A. Self care interventions to advance health and wellbeing: a conceptual framework to inform normative guidance. BMJ 2019; 365:1688 doi: $10.1136 /$ bmj.1688.

\section{Contribución de autores}

Leonel Briozzo, https://orcid.org/0000-0002-6772-3188. Concepción, diseño, ejecución, análisis, interpretación de los resultados, redacción, revisión crítica.

Fernanda Nozar, https://orcid.org/0000-0003-2820-2141. Diseño, ejecución, análisis, interpretación de los resultados, redacción, revisión crítica.

Verónica Fiol, https://orcid.org/0000-0002-4808-9302. Diseño, ejecución, análisis, interpretación de los resultados, redacción, revisión crítica.

Cecilia Stapf, https://orcid.org/0000-0002-6460-1046. Ejecución, análisis, interpretación de los resultados, redacción, revisión crítica. Sebastián Ben, https://orcid.org/000-0001-7664-4687. Ejecución, análisis, interpretación de los resultados, redacción, revisión crítica. Estela Citrin, https://orcid.org/0000-0003-4551-5107. Ejecución, análisis, interpretación de los resultados, redacción, revisión crítica. Diego Greif, https://orcid.org/0000-0003-1332-4314. Ejecución, análisis, interpretación de los resultados, redacción, revisión crítica. Verónica Gallino, https://orcid.org/0000-0002-6682-0330. Ejecución, análisis, interpretación de los resultados, redacción, revisión crítica. 